\title{
COMMUTATIVITY THEOREMS FOR GROUPS AND SEMIGROUPS
}

\author{
FRANCISCO ARAÚJO AND MICHAEL KINYON*
}

\begin{abstract}
In this note we prove a selection of commutativity theorems for various classes of semigroups. For instance, if in a separative or completely regular semigroup $S$ we have $x^{p} y^{p}=y^{p} x^{p}$ and $x^{q} y^{q}=y^{q} x^{q}$ for all $x, y \in S$ where $p$ and $q$ are relatively prime, then $S$ is commutative. In a separative or inverse semigroup $S$, if there exist three consecutive integers $i$ such that $(x y)^{i}=x^{i} y^{i}$ for all $x, y \in S$, then $S$ is commutative. Finally, if $S$ is a separative or inverse semigroup satisfying $(x y)^{3}=x^{3} y^{3}$ for all $x, y \in S$, and if the cubing map $x \mapsto x^{3}$ is injective, then $S$ is commutative.
\end{abstract}

\section{INTRODUCTION}

Broadly speaking, a commutativity theorem in group theory is any result concluding that a group is commutative, i.e. abelian. Perhaps the best known example is the following standard exercise, usually given to students at the beginning of their study of group theory:

If $G$ is a group satisfying $x^{2}=1$ for all $x \in G$, then $G$ is commutative.

Commutativity theorems can sometimes be extended to various classes of semigroups properly containing groups. For instance, a semigroup $S$ is cancellative if it satisfies the conditions $x y=x z \Longrightarrow y=z$ and $y x=z x \Longrightarrow y=z$ for all $x, y, z$. Every finite cancellative semigroup is a group; the positive integers under addition provide an example of a cancellative semigroup which is not a group.

The exercise above extends easily to cancellative semigroups once we reinterpret the condition " $x^{2}=1$ ". Since we do not wish to assume the existence of an identity element, we replace the condition with " $x^{3}=x$ ", which is clearly equivalent to $x^{2}=1$ in groups.

Proposition 1.1. If $S$ is a cancellative semigroup satisfying $x^{3}=x$ for all $x \in S$, then $S$ is commutative (and in fact, is a group satisfying $x^{2}=1$ for all $x \in S$ ).

The parenthetical part of the assertion suggests the proof: if $x^{3}=x$, then $x^{3} y=x y$ and so cancelling gives $x^{2} y=y$ for all $x, y \in S$. Dually, $y x^{2}=y$ for all $x, y \in S$. Thus for all $x, y \in S, x^{2}=x^{2} y^{2}=y^{2}$. This constant, which we denote by 1 , is an identity element. Hence $x^{3}=x=x 1$, and cancelling gives $x^{2}=1$ for all $x \in S$. Therefore $S$ is a group and we have reduced the problem to the original exercise.

Still using this elementary example to illustrate our point, further extensions of the result are possible. A semigroup is separative if it satisfies the conditions $x y=x x \& y x=y y \Longrightarrow$ $x=y$ and $x y=y y \& y x=x x \Longrightarrow x=y$ ([7], Def. II.6.2, p. 51). Every cancellative semigroup is evidently separative.

We also need the notion of a semilattice of semigroups. A semilattice is a partially ordered set $(I, \leq)$ such that every two elements $x, y \in S$ have a greatest lower bound, denoted by

\footnotetext{
* Partially supported by Simons Foundation Collaboration Grant 359872 and by FCT project CEMATCIÊNCIAS UID/Multi/04621/2013.
} 
$x \wedge y$. A semigroup $S$ is a semilattice of semigroups if there exist a semilattice $(I, \leq)$ and a set $Y=\left\{S_{\alpha}\right\}_{\alpha \in I}$ of pairwise disjoint subsemigroups $S_{\alpha} \leq S$ indexed by $I$ such that $S=\cup_{\alpha \in I} S_{\alpha}$, and satisfying this property: for all $\alpha, \beta \in I$ and for all $a \in S_{\alpha}, b \in S_{\beta}$, we have $a b \in S_{\alpha \wedge \beta}$. (For details, see [7], Def. II.1.4, p. 27).

For our purposes, the following results are key ([7, Thm. II.6.4, p. 51], [6, Thm. 3.12, p. 47]).

Proposition 1.2. Let $S$ be a semigroup.

(1) $S$ is separative if and only if $S$ is semilattice of cancellative semigroups.

(2) $S$ is commmutative and separative if and only if $S$ is a semilattice of commutative cancellative semigroups.

Now we can extend the original exercise even further.

Corollary 1.3. Let $S$ be a separative semigroup satisfying $x^{3}=x$ for all $x \in S$. Then $S$ is commutative, and in fact, is a semilattice of abelian groups satisfying $x^{2}=1$.

Indeed, by Proposition 1.2(1), $S$ is a semilattice of cancellative semigroups $S_{\alpha}, \alpha \in I$, such that each $S_{\alpha}$ satisfies $x^{3}=x$. By Proposition 1.1, each $S_{\alpha}$ is an abelian group satisfying $x^{2}=1$. By Proposition [1.2(2), $S$ is commutative.

We can also view the generalizations of our exercise from a different perspective. A semigroup $S$ is regular if for each $a \in S$, there exists $b \in S$ such that $a b a=a$. This is equivalent to asserting that each $a \in S$ has an inverse $a^{\prime} \in S$ satisfying $a a^{\prime} a=a$ and $a^{\prime} a a^{\prime}=$ $a^{\prime}$. If each $a \in S$ has a unique inverse, then $S$ is said to be an inverse semigroup. Equivalently, an inverse semigroup is precisely a regular semigroup in which all the idempotents commute. A semigroup $S$ such that each element has a commuting inverse $a a^{\prime}=a^{\prime} a$ is said to be completely regular. Equivalently, a completely regular semigroup is a union of groups. A completely regular, inverse semigroup is called a Clifford semigroup. A Clifford semigroup is characterized as a semilattice of groups. (For further details on regular semigroups, see, for instance, [3.) Note in particular that every Clifford semigroup is separative.

This gives us a different way of viewing our exercise. A semigroup satisfying $x^{3}=x$ for all $x$ is completely regular, with the commuting inverse of each $x$ being given by $x^{\prime}=x$. It is easy to see that a regular, cancellative semigroup is a group by essentially the same argument as above: $x x^{\prime} x y=x y$ and $y x x^{\prime} x=y x$, so $x^{\prime} x y=y$ and $y x x^{\prime}=y$ for all $x, y \in S$. A semigroup (or more generally, any magma) with both a left identity element and a right identity element has a (necessarily unique) identity element 1 , and we have $x x^{\prime}=x^{\prime} x=1$ for all $x \in S$. Thus we have another proof of Proposition 1.2 .

In this paper, we will extend three commutativity theorems from group theory to semigroups. Our first result, which was our original motivation, is based on a recent preprint of Venkataraman [8]. She proved that in a finite groups, if squares commute with squares and cubes commute with cubes then the group is commutative; she also proposed the problem of extending her result to infinite groups. More generally, in the same paper, she asked if a group satisfying the conditions $x^{p} y^{p}=y^{p} x^{p}$ and $x^{q} y^{q}=y^{q} x^{q}$ for all $x$ where $p$ and $q$ are relatively prime is necessarily commutative. Although we did not know it when we began our investigation, this is apparently a folk result in group theory [9], although we have not been able to find a reference in the literature. (Note that the proofs given in the cited website do 
not generalize directly to semigroups.) In the spirit of our discussion above, we prove that Venkataraman's desired result holds more generally.

Theorem 1.4. Let $S$ be a separative or completely regular semigroup such that, for all $x, y \in S, x^{p} y^{p}=y^{p} x^{p}$ and $x^{q} y^{q}=y^{q} x^{q}$ where $p$ and $q$ are relatively prime positive integers. Then $S$ is commutative.

In Example 2.2, we note that this theorem cannot be extended from completely regular semigroups to general regular semigroups.

It is easy to see that a group, or more generally a cancellative semigroup, is commutative if and only if $(x y)^{2}=x^{2} y^{2}$ for all $x, y$. The direct implication is trivial; for the converse, $x y x y=x x y y$ implies $y x=x y$ after cancellation. In the same vein is the following well-known exercise ([2], §2.3, Exer. 4):

If $G$ is a group such that $(a b)^{i}=a^{i} b^{i}$ for three consecutive integers $i$ for all $a, b \in G$, show that $G$ is abelian.

The slightly awkward wording allows two interpretations: that the integers $i$ depend on the elements $a, b$, or that the same integers $i$ work for all $a, b$. The proof for groups is essentially the same in either case. Our generalizations require both readings.

Theorem 1.5. Let $S$ be a semigroup.

(1) Suppose $S$ is separative and suppose that for each $a, b \in S$, there exist three consecutive nonnegative integers $i$ such that $(a b)^{i}=a^{i} b^{i}$. Then $S$ is commutative.

(2) Suppose $S$ is an inverse semigroup and suppose that there exist three consecutive nonnegative integers $i$ such that $(a b)^{i}=a^{i} b^{i}$ for all $a, b \in S$. Then $S$ is commutative.

Part (2) of this theorem cannot be formulated in the same way as part (1) is; see Example 3.2. In addition, part (2) cannot be generalized to other types of regular semigroups; see Example 3.3 .

Another commutativity theorem for groups was motivated for us by another known exercise [2, Exer. 24, p. 48]:

Let $G$ be a finite group whose order is not divisible by 3 . Suppose that $(a b)^{3}=a^{3} b^{3}$ for all $a, b \in G$. Prove that $\mathrm{G}$ must be abelian.

The finiteness is not essential and the condition can be replaced with the assumption that $G$ is a group with no elements of order 3, that is, $G$ satisfies the condition

$$
x^{3}=1 \Longrightarrow x=1
$$

for all $x \in G$. More generally, a group $G$ satisfying $(a b)^{3}=a^{3} b^{3}$ for all $a, b \in G$ can be described by a more general theorem of Alperin [1] as a quotient of a subgroup of a direct product of abelian groups and groups of exponent 3. The condition (1) rules out groups of exponent 3, and so $G$ is abelian. The existence of nonabelian groups of exponent 3 , such as the unique one of order 27, shows that some additional hypothesis like (1) is needed to conclude commutativity.

There are two reasonable reformulations of (1) for semigroups. First, if a semigroup $S$ satisfies $(a b)^{3}=a^{3} b^{3}$ for all $a, b \in S$, then this just asserts that the cubing mapping $S \rightarrow S ; x \mapsto x^{3}$ is an endomorphism. From this point of view, (1) asserts that in groups, the kernel of this endomorphism is trivial, or equivalently, that the endomorphism is injective. 
This latter formulation makes sense in any semigroup $S$ :

$$
x^{3}=y^{3} \Longrightarrow x=y
$$

for all $x \in S$.

Another reformulation of (11) for groups which works for any semigroup $S$, possibly without an identity element, is weaker, but more straightforward:

$$
x^{4}=x \Longrightarrow x^{2}=x
$$

for all $x \in S$. To see that this is weaker, suppose (2) holds and $x^{4}=x$. Then $\left(x^{2}\right)^{3}=x^{6}=x^{3}$, and so applying (2) yields $x^{2}=x$.

Theorem 1.6. Let $S$ be a semigroup satisfying $(x y)^{3}=x^{3} y^{3}$ for all $x \in S$.

(1) If $S$ is separative and satisfies (2), then $S$ is commutative.

(2) If $S$ is an inverse semigroup and satisfies (3), then $S$ is commutative.

The hypothesis of part (1) of Theorem 1.6 cannot be weakened to (3); see Example 4.2 below. Also, neither part of the theorem extends to other types of regular semigroups; see Example 4.3 .

All of our investigations were aided by the automated deduction tool Prover9 created by McCune [4]. Automated theorem provers are especially good at equational reasoning, being able to derive consequences of equational axioms much faster and more efficiently than humans. Any currently available automated theorem prover would have sufficed for this project, but Prover9 has the advantage that its input and output are easily readable by mathematicians with no familiarity with such tools. For example, here are the axioms in an input file for the special case of Theorem 1.4 where $S$ is a group, $p=2$ and $q=3$ :

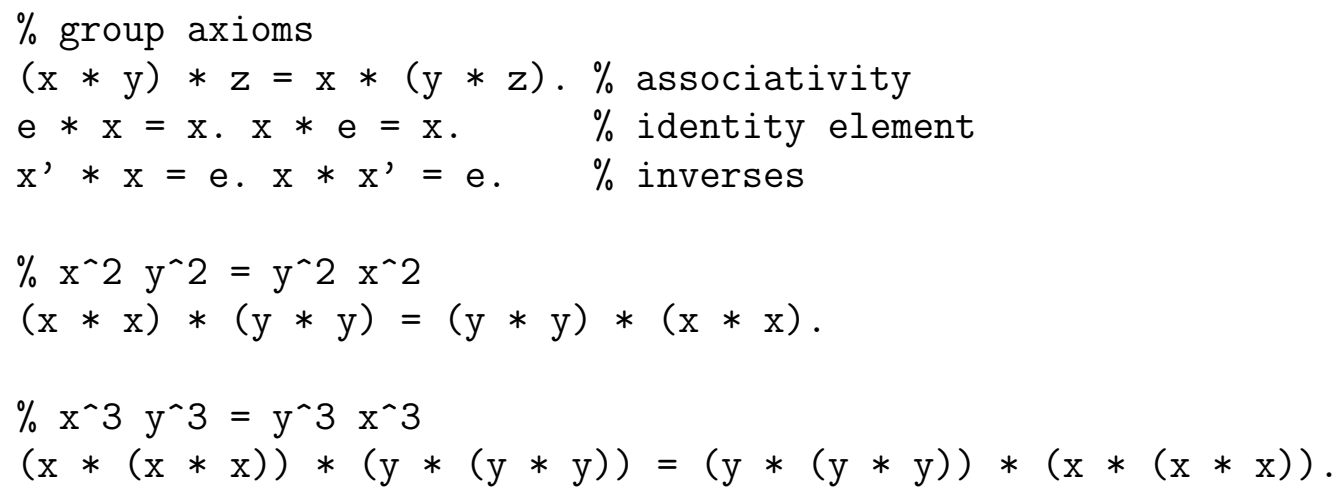

The goal is just

$\mathrm{x} * \mathrm{y}=\mathrm{y} * \mathrm{x} . \%$ commutativity

Here each equation is interpreted by Prover 9 to be universally quantified in the variables. Everything written after a \% symbol is a comment. Notice that the association of terms in any equation is made explicit; while there are settings in Prover9 which allow one to avoid parenthesization of the input, they do not generally improve readability of proofs.

Since we are working in semigroups, the associative law is part of any input file, and is heavily used throughout proofs, mostly as a rewrite rule. While this can lengthen proofs, it actually causes little to no trouble for a human reader, who can skip many lines where the rule is being applied. 
Running Prover9 with its default settings on the above input gives a proof in less than half a minute on a not particularly fast computer. The proof has 174 steps. Some of the steps are long and unpleasant; for instance, the longest one has an equation with 67 symbols in it (variables and occurrences of the operations $*$ and '. Here is a more typical one from the proof, split into two lines:

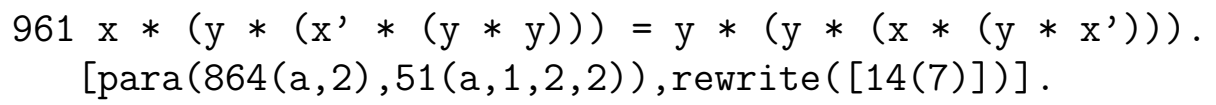

The number 961 is a clause identification number which is an internal index that Prover 9 uses to keep track of kept clauses. The part in square brackets is the justification for clause 961. Here "para" is short for paramodulation, which is the primary inference used in equational reasoning. Paramodulation refers to the substitution of one side of an equation into a subterm of another equation. In this case, clause 864 was plugged into a subterm of clause 51. This was followed by a rewrite of the resulting clause by clause 14 .

It is not terribly enlightening to show the details of this particular step in the proof nor any other step, because it turns out not to be necessary for translation into humanly readable form. A proof of 167 steps is, by the standards of automated theorem provers, not very long, and so it is reasonable to try to obtain a human proof. For familiar associative structures such as groups, lattices, rings, and so on, this is usually easy, albeit sometimes time-consuming. Given two equations and being told that under the axioms of group theory, the two yield a third is usually enough for someone familiar with groups to see how the proof goes.

In addition, a human reader can take numerous shortcuts. For example, here is another step in the proof, omitting the justification:

$11605 \mathrm{x} *\left(\mathrm{y} *\left(\mathrm{x} * \mathrm{y}^{\prime}\right)\right)=\mathrm{x} * \mathrm{x}$.

A human reader can immediately see that the proof is essentially finished: cancel $x$ on each side of the identity and then multiply on the right by $y$ to get commutativity. Prover9, on the other hand, took 14 additional steps to reach commutativity. In other words, the "out of the box" proof that Prover9 found was far from optimal.

Experienced users can tweak Prover9's many parameters and use various specialized techniques to find proofs faster, to find shorter proofs, and so on. For example, changing the term ordering from the default lexicographic path ordering (LPO) to the Knuth-Bendix ordering gets a different proof in just 10 seconds and the new proof is 19 steps shorter than the first one.

Certainly the most interesting use of Prover9 was in our investigation of Theorem 1.4. Conditions such as $x^{p} y^{p}=y^{p} x^{p}$ for all $x, y$ where $p$ is an arbitrary but fixed positive integer cannot be directly encoded in Prover9 (or any other first-order theorem prover) because it has no built-in description of the integers. Instead, we had to look at several special cases such as the one above.

It was only after examination of several special cases that we realized that many of the steps in the proofs were similar. This enabled us to see the pattern of the proof of Theorem 1.4 for groups. (Recall that at this point, we were not aware that the theorem was a known folk result.) In particular, the special cases led us to our formulation of Lemma 2.1 below as containing the essential idea of the proofs. It was also at this same point in our investigations that we realized the theorem holds more generally in cancellative, and then separative semigroups. This motivated us to look at a sampling of other commutativity theorems in various classes of semigroups. 
After this paper was submitted, we became aware of the recently published paper of Moghaddam and Padmanabhan [5]. The results contained therein are different from ours, but the spirit of the work is exactly the same: by extracting the essential features of syntactic proofs of commutativity theorems for groups, they were able to extend them to cancellative semigroups.

\section{Proof of Theorem 1.4}

The goal of this section is to prove Theorem 1.4. We start with the following key lemma.

Lemma 2.1. Let $S$ be a cancellative semigroup and suppose that there exists a map $g: S \rightarrow S$ satisfying the following conditions: for all $x, y \in S$,

(a) $x g(x)=g(x) x$;

(b) $g(x) g(y)=g(y) g(x)$;

(c) $x g(x) \cdot y g(y)=y g(y) \cdot x g(x)$.

Then the semigroup $S$ is commutative.

Proof. We claim that the following identity holds:

$$
g(g(x) y) y=y g(g(x) y)
$$

In fact,

$$
\begin{aligned}
\underbrace{g(x) y}_{u} \underbrace{g(g(x) y)}_{g(u)} & \stackrel{(a)}{=} \underbrace{g(g(x) y)}_{g(u)} \underbrace{g(x) y}_{u} \\
& =\underbrace{g(g(x) y)}_{g(u)} \underbrace{g(x)}_{g(x)} y \\
& \stackrel{(b)}{=} \underbrace{g(x)}_{g(x)} \underbrace{g(g(x) y)}_{g(u)} y
\end{aligned}
$$

which, eliminating $g(x)$ by left cancellation, gives (4).

Our next claim is that $g(x) y=y g(x)$. We start by observing that

$$
y g(y) \underbrace{g(x) y}_{u} \underbrace{g(g(x) y)}_{g(u)} \stackrel{(c)}{=} \underbrace{g(x) y}_{u} \underbrace{g(g(x) y)}_{g(u)} y g(y) .
$$


Now,

$$
\begin{aligned}
g(x) y \underbrace{g(y) g(g(x) y)}_{g(y) g(u)} y & \stackrel{(b)}{=} g(x) y \underbrace{g(g(x) y) g(y)}_{g(u) g(y)} y \\
& =g(x) y g(g(x) y) \underbrace{g(y) y} \\
& \stackrel{(a)}{=} g(x) y g(g(x) y) \underbrace{y g(y)} \\
& \stackrel{(5)}{=} y g(y) g(x) y g(g(x) y) \\
& \stackrel{(b)}{=} y \underbrace{g(x) g(y)} y g(g(x) y) \\
& \stackrel{4}{=} y g(x) g(y) \underbrace{g(g(x) y) y}
\end{aligned}
$$

yielding

$$
g(x) y \cdot(g(y) g(g(x) y) y)=y g(x) \cdot(g(y) g(g(x) y) y),
$$

which, by right cancellation, implies $g(x) y=y g(x)$, as claimed.

Now the proof that $x y=y x$ is straightforward:

$$
x \underbrace{y g(x)} g(y)=x \underbrace{g(x) y} g(y) \stackrel{(c)}{=} y g(y) x g(x)=y \underbrace{x g(y)} g(x) \stackrel{(b)}{=} y x g(x) g(y),
$$

and $x y=y x$ follows by right cancellation. This completes the proof of the lemma.

The previous lemma opens the gate to the proof of our first main theorem.

Proof of Theorem 1.4. We may assume without loss of generality that $p, q>1$.

Suppose first that $S$ is cancellative. Since $p$ and $q$ are relatively prime, by Bezout's identity there exist integers $r, s$ such that $p r+q s=1$. Since one of $p r$ or $q s$ must be negative, we assume without loss of generality that $q s<0$; thus $-q s>0$ and $x^{-q s} \in S$ for all $x \in S$. Since $q>0$, we have $s<0$ so that $-s>0$; thus $x^{-s} \in S$ for all $x \in S$. As $p r>0$ and $p>0$, we have $r>0$ and $x^{p r}, x^{r} \in S$ for all $x \in S$.

Let $g(x)=x^{-q s}$. We claim that $g(x)$ satisfies the three properties (a), (b) and (c) of the previous lemma. By associativity, we have $x g(x)=g(x) x$ so that (a) holds. Regarding (b) we have

$$
g(x) g(y)=x^{-q s} y^{-q s}=\left(x^{-s}\right)^{q}\left(y^{-s}\right)^{q}=\left(y^{-s}\right)^{q}\left(x^{-s}\right)^{q}=y^{-q s} x^{-q s}=g(y) g(x) .
$$

The second equality holds because by assumption the $q$ th powers commute. Finally, regarding (c) we have

$$
\begin{aligned}
x g(x) y g(y) & =x x^{-q s} y y^{-q s}=x^{1-q s} y^{1-q s}=x^{p r} y^{p r} \\
& =\left(x^{r}\right)^{p}\left(y^{r}\right)^{p}=\left(y^{r}\right)^{p}\left(x^{r}\right)^{p}=y^{p r} x^{p r} \\
& =y^{1-q s} x^{1-q s}=y y^{-q s} x x^{-q s}=y g(y) x g(x) .
\end{aligned}
$$

The fourth equality holds because $p$ th powers commute.

We have proved that $S$ admits a function $g: S \rightarrow S$ satisfying the three conditions of the previous lemma. It follows that $S$ is commutative. 
Next assume that $S$ is separative. By Proposition 1.2, $S$ is a semilattice of cancellative semigroups $S_{\alpha}$, each of which satisfies the hypotheses of the theorem. It follows that each $S_{\alpha}$ is commutative. By Proposition 1.2 again, $S$ is commutative.

Finally, assume that $S$ is completely regular. If $e, f \in S$ are idempotents, then $e f=$ $e^{p} f^{p}=f^{p} e^{p}=f e$. It follows that $S$ is an inverse semigroup. Since $S$ is both completely regular and inverse, it is a Clifford semigroup, hence is a semilattice of groups. In particular, $S$ is separative and the desired result follows.

Example 2.2. Theorem 1.4 does not generalize from completely regular semigroups to other types of regular semigroups. For example, let $S$ be the Brandt semigroup of order 5 . Then for every positive integer $p$ and every $x \in S, x^{p}$ is an idempotent. Thus the hypotheses of the theorem are satisfied since idempotents commute in inverse semigroups, but $S$ is not commutative.

\section{Proof of Theorems 1.5}

We first need a lemma which will prove useful in both this section and the next.

Lemma 3.1. Let $S$ be an inverse semigroup and suppose there exists an integer $k>1$ such that $(x y)^{k}=x^{k} y^{k}$ for all $x \in S$. Then $S$ is a Clifford semigroup.

Proof. Denote the unique inverse of an element $x \in S$ by $x^{\prime}$. We will show that $x x^{\prime}=x^{\prime} x$ for all $x \in S$. It will follow that $S$ is completely regular, hence Clifford. First, since $\left(x^{\prime} x\right)^{k}=x^{\prime} x$, we have

$$
\left(x^{\prime}\right)^{k} x^{k}=x^{\prime} x
$$

for all $x \in S$. Next, recalling that $\left(x^{\prime}\right)^{k-1}=\left(x^{k-1}\right)^{\prime}$ in inverse semigroups, we compute

$$
\left(x^{\prime}\right)^{k-1} x^{k}=\left(x^{k-1}\right)^{\prime} x^{k}=\underbrace{\left(x^{\prime}\right)^{k-1} x^{k-1} \cdot x x^{\prime}} x=x x^{\prime} \cdot\left(x^{\prime}\right)^{k-1} x^{k-1} x=x\left(x^{\prime}\right)^{k} x^{k} \stackrel{\underline{\underline{6}}}{=} x x^{\prime} x=x,
$$

where we used the fact that idempotents commute in the third equality. Thus

$$
\left(x^{\prime}\right)^{k-1} x^{k}=x .
$$

Next, we have

$$
x^{\prime} x x \stackrel{(7)}{=} x^{\prime} x\left(x^{\prime}\right)^{k-1} x^{k}=\left(x^{\prime}\right)^{k-1} x^{k} \stackrel{(7)}{=} x,
$$

where we used $k>1$ in the second equality. Thus we have both

$$
\begin{aligned}
x^{\prime} x x & =x, \\
x x^{\prime} x^{\prime} & =x^{\prime},
\end{aligned}
$$

where (9) follows from (8) by replacing $x$ with $x^{\prime}$ and using $x^{\prime \prime}=x$. Finally, we compute

$$
x x^{\prime} \stackrel{(8)}{=} x^{\prime} x x x^{\prime}=x x^{\prime} x^{\prime} x \stackrel{(9)}{=} x^{\prime} x,
$$

where we used commuting idempotents in the second equality. This completes the proof of the lemma.

Proof of Theorem 1.5. We begin with part (1) and suppose first that $S$ is cancellative. One of the standard proofs of Herstein's exercise ([2], §2.3, Exer. 4) only uses cancellation and so applies here. Say that the three consecutive nonnegative integers are $i, i+1, i+2$. Then $a^{i} b^{i} a b=(a b)^{i} a b=(a b)^{i+1}=a^{i+1} b^{i+1}$. Cancel $a^{i}$ on the left and $b$ on the right to get 
$b^{i} a=a b^{i}$. Repeating the same argument with $i+1$ in place of $i$ gives $b^{i+1} a=a b^{i+1}$. Thus $b^{i} \cdot a b=a b^{i} b=a b^{i+1}=b^{i+1} a=b^{i} \cdot b a$. Cancelling gives $a b=b a$.

Now suppose $S$ is separative. By Proposition 1.2, $S$ is a semilattice of cancellative semigroups $S_{\alpha}$. Each $S_{\alpha}$ satisfies the hypothesis of the theorem, hence is commutative. By Proposition 1.2, $S$ is commutative.

Now we turn to part (2) and assume that $S$ is an inverse semigroup satisfying the hypotheses of the theorem. Applying Lemma 3.1 with $k=i+1$, we have that $S$ is a Clifford semigroup. In particular, $S$ is separative. Since the hypotheses of part (2) are stronger than those of part (1), we may now apply part (1) to conclude that $S$ is commutative.

Example 3.2. Let $S$ be any nonClifford inverse semigroup with a zero 0, for instance, the Brandt semigroup of order 5 will suffice. Let $b$ be any element such that $b b^{\prime} \neq b^{\prime} b$. Then $(0 b)^{k}=0^{k} b^{k}$ for all $k \geq 1$. This shows that part (2) of Theorem 1.5 cannot be strengthened to be like part (1).

Example 3.3. Let $S=\{e, f\}$ be the 2-element left (say) zero semigroup. Then trivially $(x y)^{k}=x^{k} y^{k}$ for all $x, y \in S$ and all positive integers $k$, but $S$ is not commutative. Thus Theorem 1.5 does not extend to arbitrary regular semigroups or even completely regular semigroups.

\section{Proof of Theorem 1.6}

We start with a lemma of some independent interest.

Lemma 4.1. Let $S$ be a cancellative semigroup satisfying $(x y)^{3}=x^{3} y^{3}$ for all $x, y \in S$. Then for all $x, y \in S$,

$$
x^{3} y=y x^{3} .
$$

Proof. First, cancellation on both sides of $(x y)^{3}=x^{3} y^{3}$ gives $(y x)^{2}=x^{2} y^{2}$ for all $x, y \in S$. Using this, we compute $(x \cdot y x)(x \cdot y x)=(y x)^{2} x^{2}=x^{2} y^{2} x^{2}$. Cancelling on both sides, we obtain

$$
x y^{2} x=y x^{2} x
$$

for all $x, y \in S$. Next, $x \cdot y^{2} x^{2} \cdot x=x(x y)^{2} x \stackrel{\text { 111 }}{=} x y \cdot x^{2} \cdot x y$. Cancelling $x y$ on the left gives $y x^{3}=x^{3} y$ for all $x \in S$, as desired.

Finally, we prove our last main result.

Proof of Theorem 1.6. For part (1), assume first that $S$ is cancellative and satisfies (2). Then (10) shows that the image $C=\left\{x^{3} \mid x \in S\right\}$ of the cubing map $S \rightarrow S ; x \mapsto x^{3}$ is commutative. The condition (2) asserts that this map is injective, hence $S$ is isomorphic to $C$. In particular, $S$ is commutative.

Now assume $S$ is separative. By Proposition [1.2, $S$ is a semilattice of cancellative semigroups $S_{\alpha}$, each of which satisfies both (21) and $(x y)^{3}=x^{3} y^{3}$ for all $x \in S_{\alpha}$. By the argument above, each $S_{\alpha}$ is commutative. Applying Proposition 1.2 again, we have that $S$ is commutative.

For (2), now let $S$ be an inverse semigroup satisfying both (3) and $(x y)^{3}=x^{3} y^{3}$ for all $x \in S$. By Lemma 3.1, $S$ is a Clifford semigroup, hence a semilattice of groups $S_{\alpha}$. Each group $S_{\alpha}$ satisfies (3) as well, but in groups, (3) is equivalent to (2). In particular, $S$ is a separative semigroup satisfying the conditions of part (1), and so $S$ is commutative. 
Example 4.2. The hypothesis of part (1) of Theorem 1.6 cannot be weakened to (3). Indeed, let $S=\left\{\left[\begin{array}{ll}1 & a \\ 0 & b\end{array}\right] \mid a, b \in \mathbb{Z}^{+}\right\}$with matrix multiplication as the operation. Then $S$ is a cancellative semigroup without idempotents and thus trivially satisfies (3) $)$. However, $S$ is not commutative.

Example 4.3. Let $S$ be as in Example 3.2 and note once again that $(x y)^{3}=x^{3} y^{3}$ is trivially satisfied for all $x, y \in S$. Since $S$ is idempotent, conditions (21) and (3) both hold. However $S$ is not commutative. Thus neither part of Theorem 1.6 extends to other types of regular semigroups.

\section{REFERENCES}

[1] J. L. Alperin, The classification of $n$-abelian groups, Canad. J. Math. 21 (1969), 1238-1244.

[2] I. N. Herstein, Topics in Algebra, Second edition. Xerox College Publishing, Lexington, Mass.-Toronto, Ont., 1975.

[3] J. M. Howie, Fundamentals of semigroup theory, London Mathematical Society Monographs. New Series, 12. Oxford Science Publications. The Clarendon Press, Oxford University Press, New York, 1995.

[4] W. W. McCune, Prover9 and Mace4, version 2009-11A. http://www.cs.unm.edu/mccune/prover9//

[5] G. I. Moghaddam and R. Padmanabhan, Commutativity theorems for cancellative semigroups, Semigroup Forum 95 (2017), 448-454.

[6] A. Nagy, Special classes of semigroups, Advances in Mathematics (Dordrecht) 1, Kluwer Academic Publishers, Dordrecht, 2001.

[7] M. Petrich, Introduction to Semigroups, Merrill Research and Lecture Series. Charles E. Merrill Publishing Co., Columbus, Ohio, 1973.

[8] G. Venkataraman, Groups in which squares and cubes commute, https://arxiv.org/abs/1605.05463.

[9] A group such that $a^{m} b^{m}=b^{m} a^{m}$ and $a^{n} b^{n}=b^{n} a^{n}$ ( $m, n$ coprime) is abelian?

https://math.stackexchange.com/questions/326702/a-group-such-that-am-bm-bm-am-and-an-bn-bn-an-m-n-

(Araújo) Colégio Planalto, R. Armindo Rodrigues 28, 1600-414 Lisboa, Portugal

(Kinyon) Department of Mathematics, University of Denver, Denver, CO 80208, USA

(Kinyon) Cemat-CiÊnCias, Departamento de Matemática, Faculdade de Ciências, UniVersidade De Lisboa, 1749-016, Lisboa, Portugal

E-mail address: mkinyon@du.edu 